\title{
Estimating Nanoscale Surface Roughness of Polyethylene Terephthalate Fibers
}

Loránd Románszki ${ }^{1}$, Szilvia Klébert ${ }^{2}$, and Károly Héberger ${ }^{2 *}$

${ }^{1}$ Functional Interfaces Research Group

${ }^{2}$ Plasma Chemistry Research Group

Institute of Materials and Environmental Chemistry, Research Centre for Natural Sciences, Hungarian Academy of Sciences, Magyar tudósok körútja 2, 1117 Budapest, Hungary

\section{Supplementary Table 1}

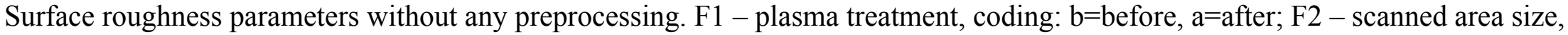
$\mu \mathrm{m}^{2}$

\begin{tabular}{cccccccccccc}
\hline & $R_{\mathrm{q}} / \mathrm{nm}$ & $R_{\mathrm{a}} / \mathrm{nm}$ & $R_{\mathrm{max}} / \mathrm{nm}$ & $R_{\mathrm{z}} / \mathrm{nm}$ & $\mathrm{peak}$ count & $R_{\mathrm{p}} / \mathrm{nm}$ & $R_{\mathrm{pm}} / \mathrm{nm}$ & $R_{\mathrm{v}} / \mathrm{nm}$ & $R_{\mathrm{vm}} / \mathrm{nm}$ & $\mathrm{F} 1$ & $\mathrm{~F} 2$ \\
\hline 1 & 6.851 & 5.304 & 77.711 & 54.12 & 72 & 29.037 & 13.793 & -48.674 & -13.177 & $\mathrm{~b}$ & 4 \\
2 & 5.344 & 4.054 & 53.374 & 42.464 & 90 & 29.792 & 11.396 & -23.582 & -10.29 & $\mathrm{~b}$ & 4 \\
3 & 4.968 & 3.702 & 50.017 & 42.044 & 84 & 27.442 & 10.132 & -22.575 & -9.279 & $\mathrm{~b}$ & 4 \\
4 & 6.612 & 5.193 & 60.843 & 53.038 & 200 & 29.372 & 12.299 & -31.47 & -13.189 & $\mathrm{~b}$ & 4 \\
5 & 5.569 & 4.401 & 64.451 & 46.66 & 212 & 31.722 & 11.75 & -32.729 & -10.46 & $\mathrm{~b}$ & 4 \\
6 & 5.782 & 4.55 & 56.982 & 46.912 & 146 & 29.288 & 11.034 & -27.694 & -11.244 & $\mathrm{~b}$ & 4 \\
7 & 10.11 & 5.694 & 116.82 & 65.962 & 7 & 68.396 & 30.236 & -48.422 & -17.738 & $\mathrm{~b}$ & 4 \\
8 & 9.043 & 5.802 & 105.32 & 60.591 & 20 & 41.708 & 17.338 & -63.612 & -19.601 & $\mathrm{~b}$ & 4 \\
9 & 10.259 & 8.117 & 97.516 & 74.102 & 103 & 47.919 & 19.211 & -49.597 & -19.106 & $\mathrm{~b}$ & 4 \\
10 & 5.775 & 4.425 & 43.555 & 36.673 & 52 & 23.414 & 11.16 & -20.141 & -10.179 & $\mathrm{~b}$ & 4 \\
11 & 4.798 & 3.69 & 49.849 & 37.513 & 56 & 24.337 & 9.633 & -25.512 & -9.034 & $\mathrm{~b}$ & 4 \\
12 & 4.78 & 3.679 & 39.359 & 35.834 & 159 & 20.309 & 9.095 & -19.05 & -9.44 & $\mathrm{~b}$ & 4 \\
13 & 8.052 & 6.438 & 58.157 & 52.199 & 82 & 26.603 & 14.382 & -31.554 & -17.255 & $\mathrm{~b}$ & 4 \\
14 & 5.748 & 4.511 & 56.647 & 42.296 & 98 & 20.057 & 10.33 & -36.589 & -11.287 & $\mathrm{~b}$ & 4 \\
15 & 7.477 & 5.658 & 65.206 & 54.632 & 120 & 35.163 & 14.146 & -30.044 & -14.132 & $\mathrm{~b}$ & 4 \\
16 & 5.401 & 4.179 & 52.283 & 42.716 & 260 & 26.603 & 11.331 & -25.68 & -10.885 & $\mathrm{~b}$ & 4 \\
17 & 6.212 & 4.868 & 55.052 & 47.415 & 165 & 29.792 & 12.016 & -25.26 & -12.136 & $\mathrm{a}$ & 4 \\
18 & 11.524 & 7.469 & 136.2 & 80.648 & 29 & 82.914 & 23.003 & -53.29 & -22.267 & $\mathrm{a}$ & 4
\end{tabular}




\begin{tabular}{|c|c|c|c|c|c|c|c|c|c|c|}
\hline 19 & 8.94 & 6.958 & 88.956 & 70.577 & 82 & 44.898 & 16.78 & -44.058 & -17.852 & $\mathrm{a}$ \\
\hline 20 & 23.803 & 12.948 & 215.76 & 154.5 & 3 & 104.48 & 84.508 & -111.28 & -70.018 & $\mathrm{a}$ \\
\hline 21 & 9.583 & 7.364 & 81.739 & 72.172 & 60 & 38.604 & 17.967 & -43.135 & -19.423 & $\mathrm{a}$ \\
\hline 22 & 7.885 & 6.157 & 66.801 & 59.836 & 49 & 24.505 & 14.962 & -42.296 & -18.243 & $\mathrm{a}$ \\
\hline 23 & 7.417 & 5.421 & 81.487 & 62.605 & 59 & 44.73 & 14.45 & -36.757 & -14.837 & $\mathrm{a}$ \\
\hline 24 & 7.267 & 5.503 & 72.004 & 56.982 & 66 & 35.757 & 14.478 & -35.247 & -15.091 & $\mathrm{a}$ \\
\hline 25 & 9.348 & 6.785 & 99.95 & 63.276 & 40 & 56.563 & 17.598 & -43.387 & -17.226 & $\mathrm{a}$ \\
\hline 26 & 6.853 & 5.419 & 62.941 & 52.115 & 99 & 29.624 & 13.66 & -33.316 & -13.308 & $\mathrm{a}$ \\
\hline 27 & 8.551 & 6.539 & 75.445 & 67.137 & 64 & 26.1 & 16.072 & -49.345 & -19.4 & $\mathrm{a}$ \\
\hline 28 & 7.638 & 5.882 & 72.004 & 63.36 & 143 & 28.785 & 14.754 & -43.219 & -15.486 & $\mathrm{a}$ \\
\hline 29 & 6.267 & 4.777 & 62.353 & 53.038 & 85 & 29.288 & 13.073 & -33.065 & -12.402 & $\mathrm{a}$ \\
\hline 30 & 20.394 & 15.297 & 217.19 & 163.39 & 86 & 102.89 & 41.616 & -114.3 & -36.891 & b \\
\hline 31 & 5.361 & 4.104 & 59.416 & 52.786 & 404 & 36.17 & 10.72 & -23.246 & -10.096 & $\mathrm{~b}$ \\
\hline 32 & 13.741 & 10.188 & 163.98 & 104.4 & 75 & 89.208 & 26.118 & -74.773 & -25.779 & b \\
\hline 33 & 14.468 & 10.241 & 148.29 & 102.72 & 54 & 66.801 & 27.431 & -81.487 & -27.055 & b \\
\hline 34 & 8.267 & 5.675 & 135.45 & 90.634 & 641 & 104.4 & 16.04 & -31.051 & -15.321 & b \\
\hline 35 & 17.303 & 9.344 & 274.59 & 143.25 & 49 & 186.98 & 35.367 & -87.613 & -32.11 & b \\
\hline 36 & 7.752 & 5.223 & 188.91 & 105.24 & 223 & 109.1 & 15.915 & -79.809 & -15.769 & $\mathrm{~b}$ \\
\hline 37 & 5.666 & 4.262 & 78.13 & 60.759 & 703 & 43.807 & 12.464 & -34.323 & -11.364 & $b$ \\
\hline 38 & 13.386 & 10.473 & 124.03 & 106.24 & 166 & 62.101 & 25.743 & -61.933 & -24.411 & b \\
\hline 39 & 11.904 & 6.526 & 249.41 & 116.23 & 383 & 97.684 & 23.052 & -151.73 & -26.103 & b \\
\hline 40 & 9.443 & 5.972 & 175.14 & 99.194 & 119 & 98.019 & 19.878 & -77.123 & -17.796 & $\mathrm{~b}$ \\
\hline 41 & 10.845 & 7.851 & 181.02 & 102.38 & 333 & 129.83 & 20.003 & -51.192 & -20.279 & $\mathrm{a}$ \\
\hline 42 & 21.476 & 11.666 & 303.71 & 184.12 & 31 & 188.07 & 45.574 & -115.64 & -48.55 & $\mathrm{a}$ \\
\hline 43 & 10.257 & 7.337 & 202 & 155.42 & 194 & 115.64 & 21.354 & -86.354 & -19.903 & $\mathrm{a}$ \\
\hline 44 & 21.171 & 13.681 & 250.17 & 125.38 & 29 & 180.93 & 41.246 & -69.235 & -37.457 & $\mathrm{a}$ \\
\hline 45 & 12.898 & 10.132 & 123.87 & 105.99 & 258 & 63.109 & 23.594 & -60.759 & -25.136 & $\mathrm{a}$ \\
\hline 46 & 8.452 & 6.595 & 80.312 & 76.871 & 170 & 29.204 & 16.002 & -51.108 & -20.299 & $\mathrm{a}$ \\
\hline 47 & 11.583 & 8.113 & 169.94 & 108.59 & 133 & 121.35 & 22.731 & -48.59 & -22.387 & $\mathrm{a}$ \\
\hline 48 & 9.543 & 7.254 & 101.46 & 88.117 & 153 & 47.835 & 20.027 & -53.625 & -22.228 & $\mathrm{a}$ \\
\hline 49 & 8.616 & 6.305 & 105.4 & 89.711 & 128 & 55.136 & 17.552 & -50.269 & -17.125 & $\mathrm{a}$ \\
\hline 50 & 24.754 & 11.338 & 345.53 & 140.74 & 8 & 55.266 & 43.283 & -290.26 & -63.707 & $\mathrm{a}$ \\
\hline
\end{tabular}




\begin{tabular}{|c|c|c|c|c|c|c|c|c|c|c|}
\hline 51 & 9.876 & 6.152 & 211.04 & 118.75 & 106 & 135.79 & 20.494 & -75.259 & -18.713 & $\mathrm{a}$ \\
\hline 52 & 9.15 & 6.515 & 129.15 & 101.96 & 247 & 68.396 & 18.012 & -60.759 & -18.251 & $\mathrm{a}$ \\
\hline 53 & 10.739 & 8.317 & 129.49 & 93.32 & 172 & 79.893 & 20.691 & -49.597 & -20.166 & $\mathrm{a}$ \\
\hline 54 & 21.845 & 14.281 & 253.11 & 158.19 & 20 & 112.2 & 42.653 & -140.9 & -42.344 & $\mathrm{a}$ \\
\hline 55 & 9.249 & 6.973 & 129.07 & 92.229 & 191 & 85.012 & 19.281 & -44.058 & -19.262 & $\mathrm{a}$ \\
\hline 56 & 13.395 & 9.978 & 152.57 & 126.47 & 274 & 88.956 & 28.456 & -63.612 & -24.352 & b \\
\hline 57 & 7.08 & 5.481 & 90.299 & 78.886 & 1493 & 53.877 & 14.25 & -36.422 & -14.33 & $b$ \\
\hline 58 & 24.119 & 16.196 & 332.41 & 181.27 & 24 & 214.92 & 50.772 & -117.49 & -48.879 & b \\
\hline 59 & 10.843 & 7.608 & 186.22 & 121.94 & 263 & 103.22 & 21.652 & -82.998 & -20.287 & b \\
\hline 60 & 12.432 & 8.336 & 192.77 & 158.78 & 202 & 113.54 & 25.971 & -79.221 & -22.175 & $\mathrm{~b}$ \\
\hline 61 & 10.807 & 6.972 & 195.54 & 127.39 & 141 & 129.15 & 26.471 & -66.381 & -19.972 & $\mathrm{~b}$ \\
\hline 62 & 22 & 13.597 & 266.95 & 160.29 & 65 & 111.45 & 41.36 & -155.51 & -38.86 & $b$ \\
\hline 63 & 30.505 & 19.539 & 389.48 & 242.7 & 86 & 219.96 & 56.332 & -169.52 & -55.165 & $\mathrm{a}$ \\
\hline 64 & 21.526 & 15.336 & 261.58 & 162.13 & 141 & 114.64 & 38.372 & -146.95 & -37.541 & $\mathrm{a}$ \\
\hline 65 & 22.05 & 15.531 & 259.15 & 152.06 & 34 & 196.12 & 46.813 & -63.024 & -38.934 & $\mathrm{a}$ \\
\hline 66 & 17.15 & 12.266 & 234.06 & 213.24 & 263 & 131.76 & 36.481 & -102.3 & -33.808 & $\mathrm{a}$ \\
\hline 67 & 15.466 & 8.643 & 273.5 & 128.82 & 31 & 186.3 & 32.342 & -87.194 & -29.905 & $\mathrm{a}$ \\
\hline 68 & 12.02 & 8.807 & 216.35 & 123.87 & 260 & 134.02 & 23.202 & -82.326 & -23.819 & $\mathrm{a}$ \\
\hline 69 & 8.409 & 6.392 & 108.17 & 90.215 & 333 & 57.737 & 16.551 & -50.436 & -16.713 & $\mathrm{a}$ \\
\hline 70 & 11.723 & 8.159 & 160.22 & 116.06 & 115 & 90.06 & 22.942 & -70.164 & -22.605 & $\mathrm{a}$ \\
\hline 71 & 15.212 & 9.934 & 284.83 & 221.55 & 118 & 134.27 & 32.287 & -150.55 & -34.077 & $\mathrm{a}$ \\
\hline 72 & 40.058 & 24.238 & 522.49 & 302.7 & 17 & 216.85 & 87.357 & -305.64 & -79.353 & $\mathrm{a}$ \\
\hline 73 & 11.714 & 8.331 & 216.85 & 129.66 & 173 & 85.431 & 25.972 & -131.42 & -23.487 & $\mathrm{a}$ \\
\hline 74 & 12.911 & 9.247 & 164.15 & 120.85 & 492 & 86.69 & 23.776 & -77.459 & -23.121 & $\mathrm{~b}$ \\
\hline 75 & 38.315 & 24.551 & 495.97 & 350.03 & 56 & 236.24 & 89.914 & -259.74 & -75.406 & $b$ \\
\hline 76 & 13.997 & 9.434 & 315.46 & 234.14 & 396 & 189.33 & 28.337 & -126.13 & -27.612 & $\mathrm{~b}$ \\
\hline 77 & 22.647 & 16.048 & 226.5 & 200.4 & 77 & 103.89 & 47.617 & -122.61 & -42.574 & $\mathrm{~b}$ \\
\hline 78 & 8.427 & 5.924 & 138.22 & 104.31 & 713 & 94.495 & 18.622 & -43.723 & -15.928 & $\mathrm{~b}$ \\
\hline 79 & 21.945 & 16.927 & 344.91 & 213.07 & 199 & 181.27 & 46.062 & -163.65 & -38.777 & $\mathrm{~b}$ \\
\hline 80 & 11.352 & 8.334 & 184.37 & 136.87 & 427 & 100.87 & 22.732 & -83.501 & -21.526 & $b$ \\
\hline 81 & 13.708 & 10.034 & 175.31 & 140.48 & 315 & 109.94 & 30.047 & -65.374 & -25.138 & $\mathrm{~b}$ \\
\hline 82 & 8.073 & 5.971 & 180.85 & 100.45 & 1481 & 87.278 & 15.93 & -93.572 & -15.822 & $\mathrm{~b}$ \\
\hline
\end{tabular}




\begin{tabular}{cccccccccccc}
83 & 17.941 & 11.051 & 313.36 & 161.88 & 59 & 217.44 & 34.123 & -95.921 & -32.662 & $\mathrm{~b}$ & 64 \\
84 & 14.318 & 9.65 & 193.1 & 151.9 & 198 & 110.19 & 29.977 & -82.914 & -26.372 & $\mathrm{~b}$ & 64 \\
85 & 14.779 & 9.794 & 248.66 & 187.65 & 186 & 163.06 & 31.64 & -85.599 & -26.474 & $\mathrm{~b}$ & 64 \\
86 & 11.975 & 8.395 & 224.82 & 148.04 & 215 & 128.15 & 25.207 & -96.677 & -22.344 & $\mathrm{~b}$ & 64 \\
87 & 41.575 & 31.244 & 329.05 & 256.88 & 96 & 150.3 & 69.068 & -178.75 & -70.231 & $\mathrm{a}$ & 64 \\
88 & 46.943 & 35.806 & 435.88 & 262.17 & 23 & 198.22 & 89.182 & -237.66 & -83.953 & $\mathrm{a}$ & 64 \\
89 & 38.612 & 20.352 & 600.03 & 310.67 & 10 & 217.1 & 101.91 & -382.93 & -99.356 & $\mathrm{a}$ & 64 \\
90 & 9.366 & 7.217 & 107.42 & 88.704 & 285 & 59.836 & 18.89 & -47.583 & -17.851 & $\mathrm{a}$ & 64 \\
91 & 20.926 & 10.594 & 353.87 & 219.7 & 23 & 70.438 & 40.663 & -283.43 & -54.82 & $\mathrm{a}$ & 64 \\
92 & 12.808 & 9.527 & 149.13 & 122.36 & 167 & 80.228 & 24.765 & -68.899 & -24.683 & $\mathrm{a}$ & 64 \\
93 & 33.318 & 22.192 & 385.87 & 258.48 & 64 & 205.1 & 62.212 & -180.77 & -65.143 & $\mathrm{a}$ & 64 \\
94 & 18.494 & 12.004 & 338.03 & 225.83 & 157 & 206.53 & 37.842 & -131.5 & -34.031 & $\mathrm{a}$ & 64 \\
95 & 10.298 & 7.674 & 138.13 & 110.61 & 263 & 80.144 & 22.723 & -57.989 & -21.244 & $\mathrm{a}$ & 64 \\
\hline
\end{tabular}


Supplementary Figure 1. Effect of plasma treatment (F1) two levels: b-before, a-after treatment. Vertical bars denote 0.95 confidence intervals. Number of samples, b: 423 and a: 432.

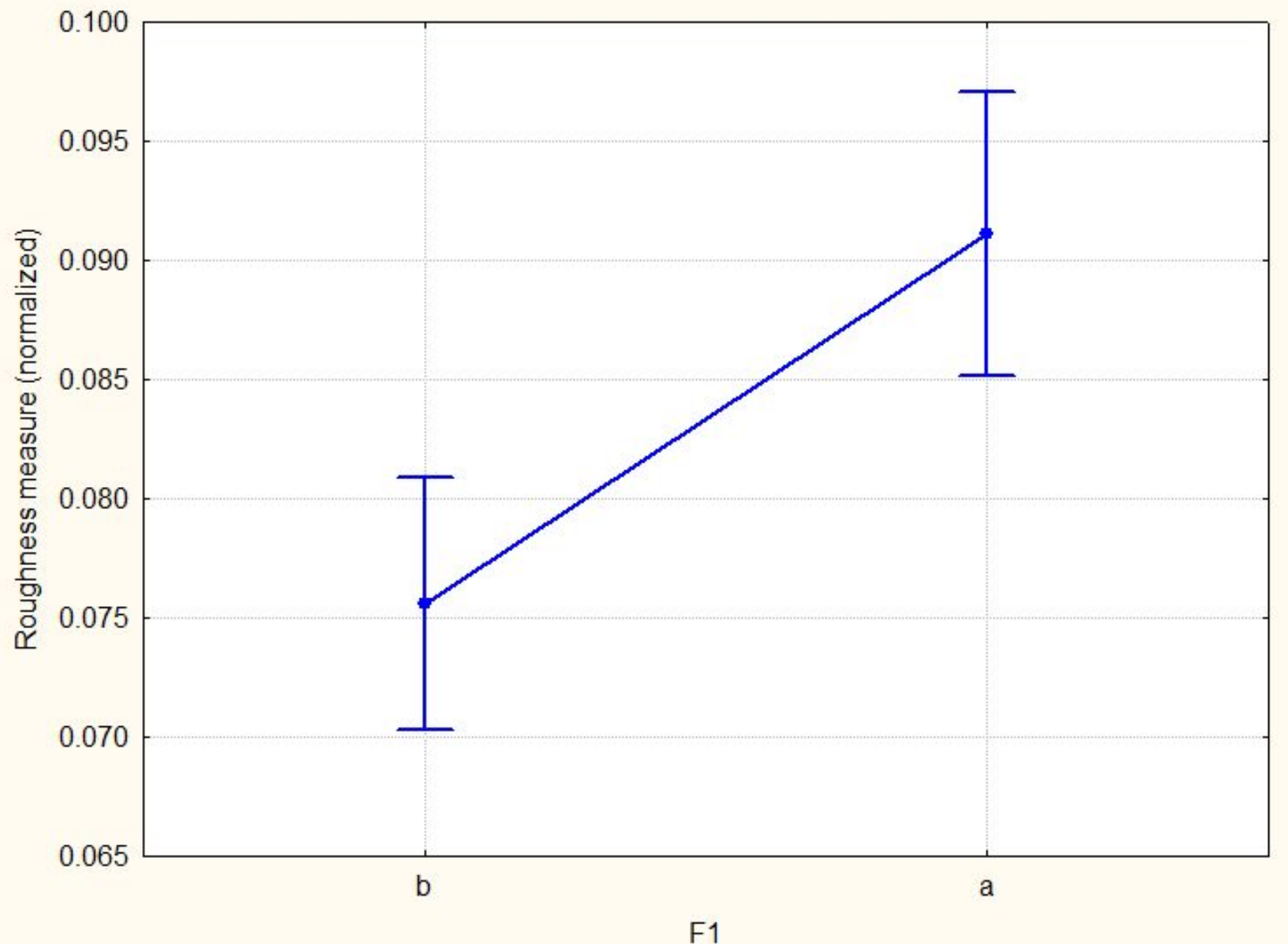

F1; Weighted Means $\mathrm{F}(1,783)=21.535, \mathrm{p}=.00000$ Effective hypothesis decomposition

\begin{tabular}{|l|l|l|l|l|l|l|}
\hline & F1 & Mean Roughness & STD Roughness & $\begin{array}{c}\text { Roughness } \\
\mathbf{- 9 5 \%}\end{array}$ & $\begin{array}{c}\text { Roughness } \\
+\mathbf{9 5 \%}\end{array}$ & $\mathbf{N}$ \\
\hline $\mathbf{1}$ & before & 0.075584 & 0.002680 & 0.070317 & 0.080852 & 423 \\
\hline $\mathbf{2}$ & after & 0.091090 & 0.003037 & 0.085122 & 0.097059 & 432 \\
\hline
\end{tabular}

i.e. the normalized roughness (independently of the way it was given) increased cca $20 \%$. 
Supplementary Figure 2. Effect of various ways to determine roughness by AFM. Vertical bars denote 0.95 confidence intervals. Notations: root mean square roughness $\left(R_{\mathrm{q}}\right)$, mean roughness $\left(R_{\mathrm{a}}\right)$, max. height $\left(R_{\max }\right)$, ten points mean $\left(R_{\mathrm{z}}\right)$, peak count, max. peak height $\left(R_{\mathrm{p}}\right)$, average max. height $\left(R_{\mathrm{pm}}\right)$, opposite of max. depth $\left(R_{\mathrm{v}}\right)$, opposite of average max. depth $\left(R_{\mathrm{vm}}\right)$

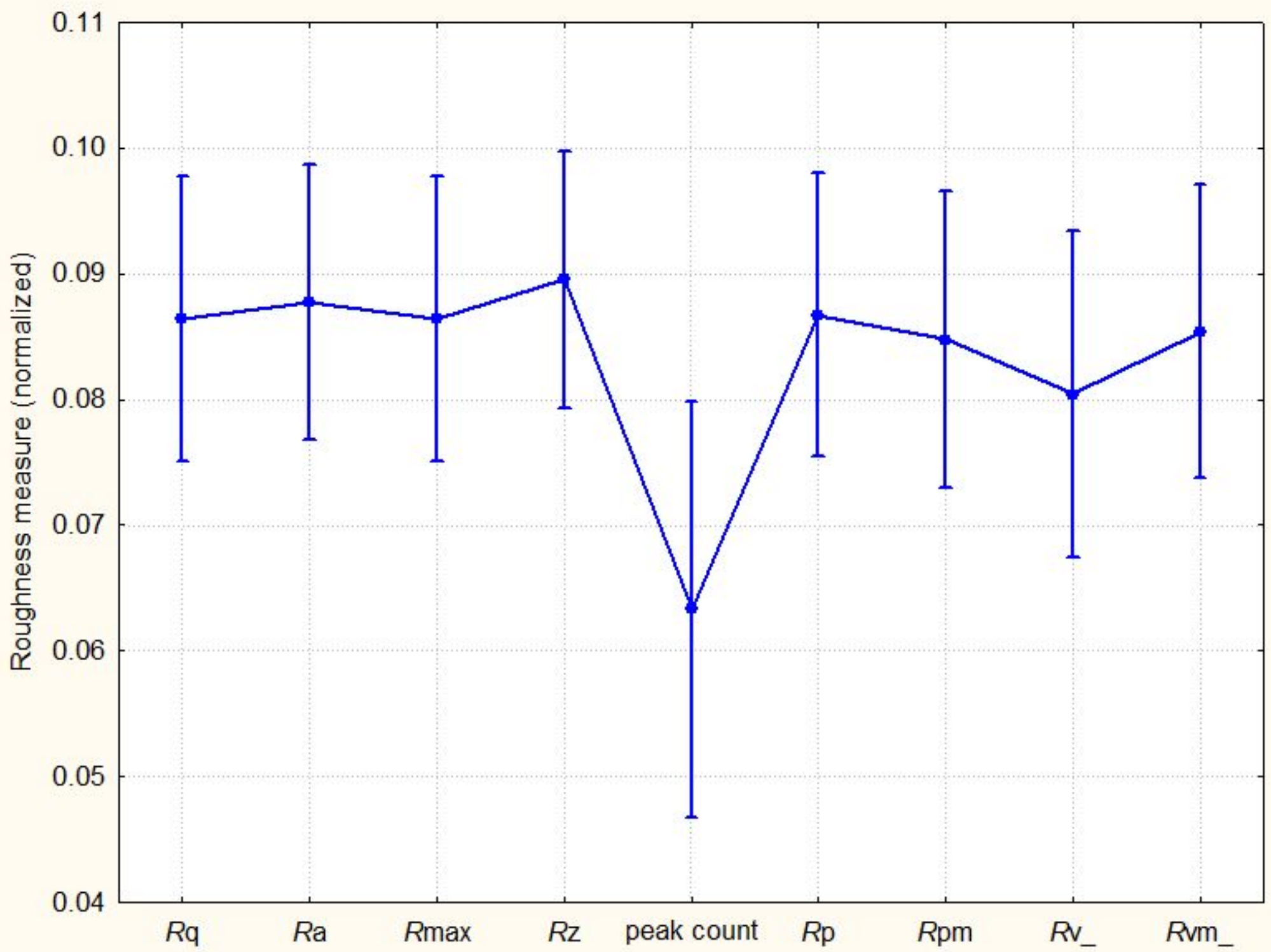


Supplementary Figure 3. Normal probability plot of residuals (Eq. (6)) showing the rudity of the modeling

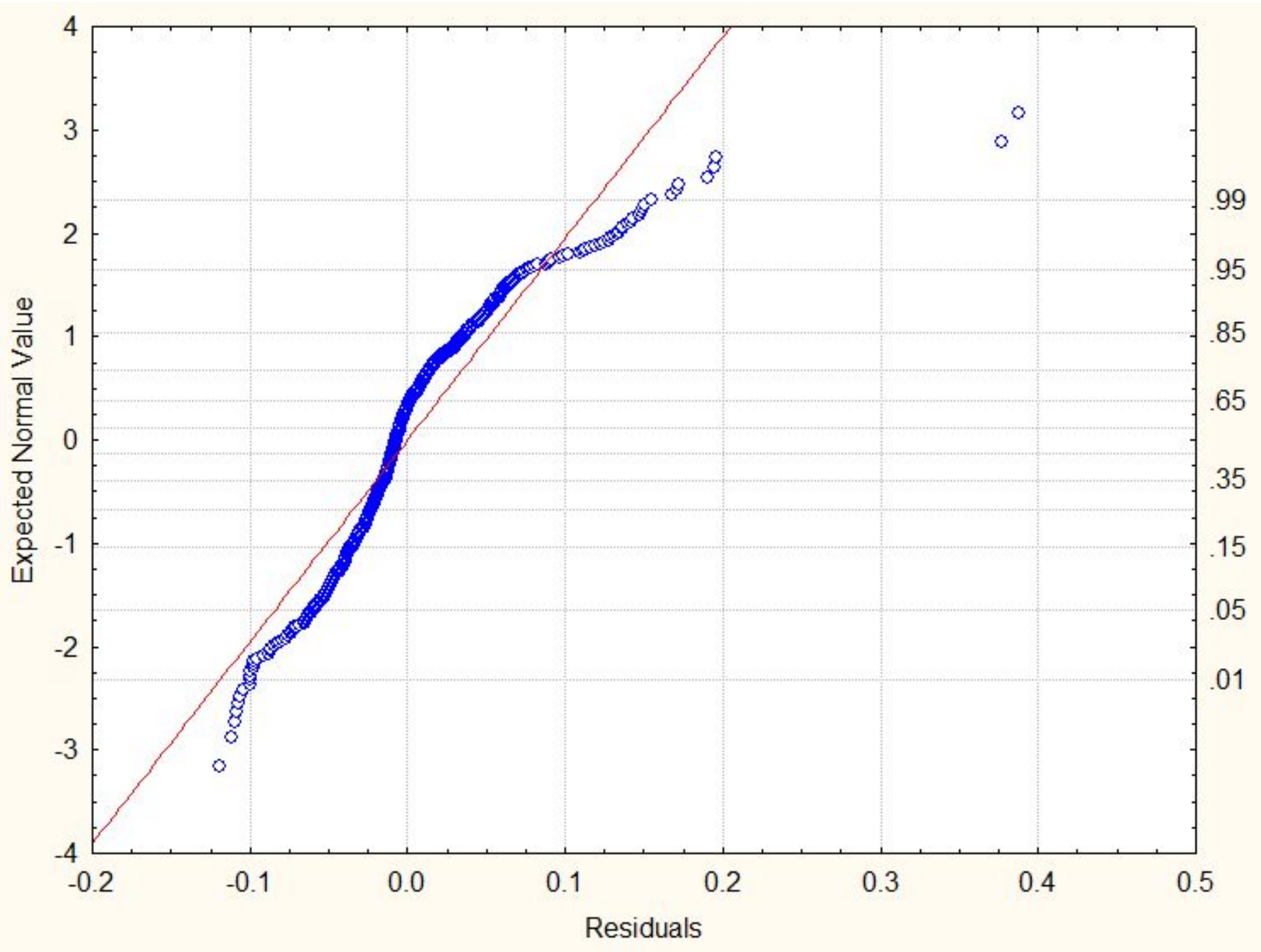


Supplementary Figure 4. Normal probability plot of residuals (Eq. (7)) showing a considerably better modeling

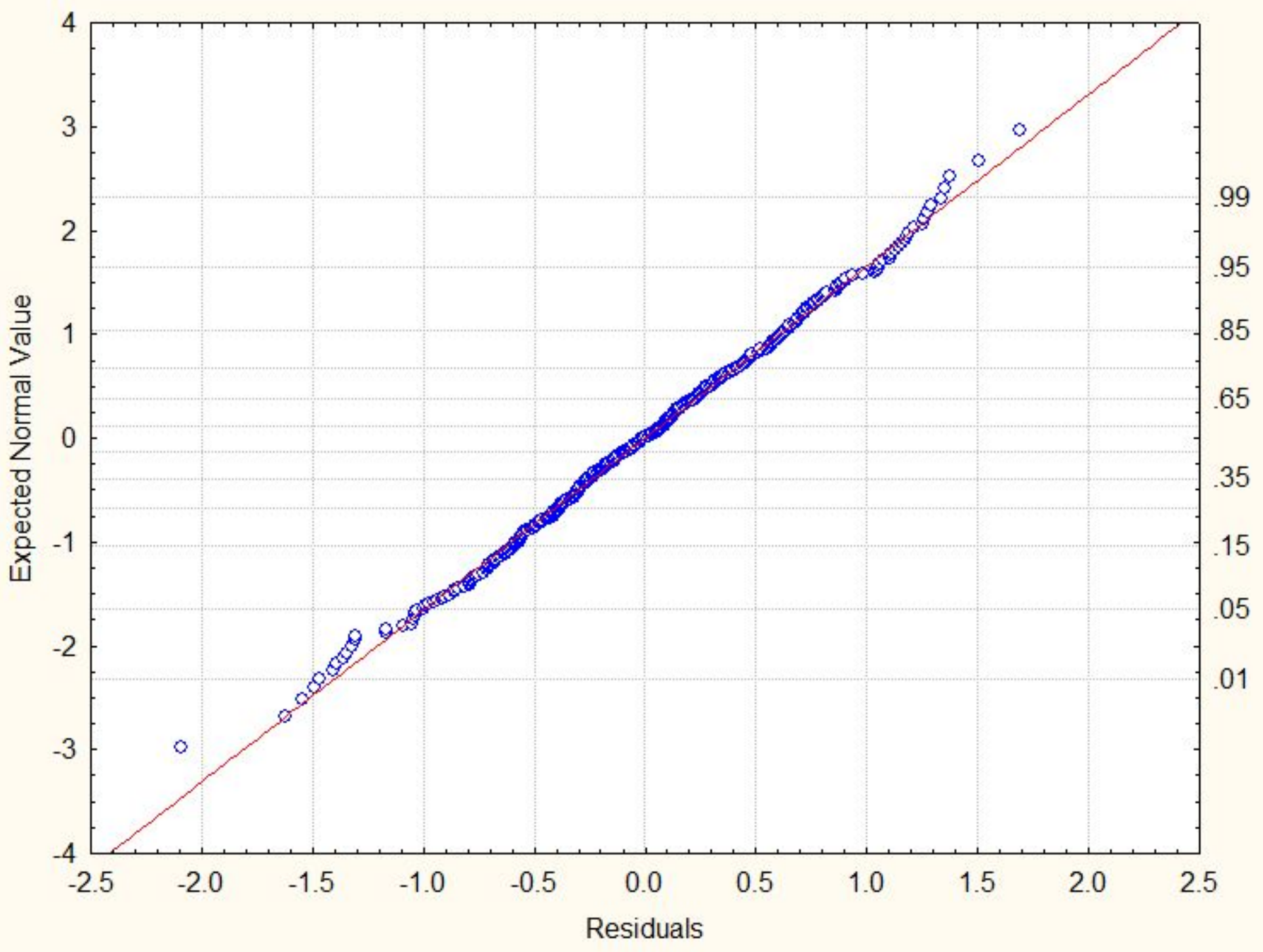

